\title{
Lost expression of cell adhesion molecule 1 is associated with bladder cancer progression and recurrence and its overexpression inhibited tumor cell malignant behaviors
}

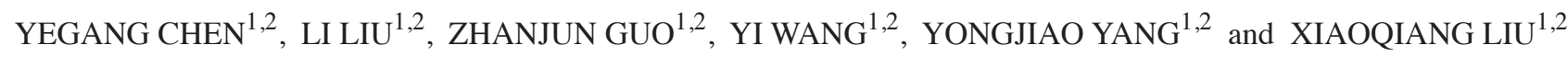 \\ ${ }^{1}$ Department of Urology, The Second Hospital of Tianjin Medical University; ${ }^{2}$ Tianjin Institute of Urology, \\ Tianjin 300211, P.R. China
}

Received September 13, 2015; Accepted March 17, 2017

DOI: $10.3892 / \mathrm{ol} .2018 .9845$

\begin{abstract}
Cell adhesion molecule 1 (CADM1) regulates cell-cell adhesion and an altered expression level is associated with tumorigenesis and progression. The present study assessed CADM1 expression level in 84 bladder tissues to investigate the association with clinicopathological parameters from bladder cancer patients and then investigated the effects of CADM1 overexpression on T24 bladder cancer cells in vitro. The results demonstrated that expression level of CADM1 protein was significantly reduced in bladder cancer tissues $(0.26 \pm 0.14)$ compared with in normal bladder mucosa $(0.69 \pm 0.092$; $\mathrm{P}<0.01$ ), and methylation of CADM1 promoter was responsible for silencing of CADM1 protein expression and significantly associated with tumor size, recurrence, pathology classification and clinical stage $(\mathrm{P}<0.05)$. Overexpression of CADM1 protein inhibited tumor cell proliferation, reduced tumor cell invasion capacity and induced tumor cell apoptosis in vitro. At the gene level, CADM1 expression level upregulated caspase-3 activity and expression of Bax and p27 protein and downregulated levels of B cell lymphoma-2, cyclinD1, cyclinE1 and cyclin dependent kinase 2 proteins. Furthermore, overexpression of CADM1 regulated the expression level of epithelial to mesenchymal transition markers, including increased expression level of E-cadherin and $\beta$-catenin, whereas it decreased the levels of Vimentin. The present study demonstrated that lost expression of CADM1 protein may exert an essential role in the development and progression of bladder cancer and suggested that CADM1 may be a novel molecular target for the control of this disease in clinical practice.
\end{abstract}

Correspondence to: Professor Xiaoqiang Liu, Department of Urology, The Second Hospital of Tianjin Medical University and Tianjin Institute of Urology, 23 Pingjiang Road, Hexi, Hebei, Tianjin 300211, P.R. China

E-mail: xiaoqiangliu1@163.com

Key words: cell adhesion molecule 1, bladder cancer, biomarker, tumor cell proliferation and invasion, epithelial to mesenchymal transition

\section{Introduction}

Bladder cancer is one of the most significant worldwide health problems, accounting for an estimated 386,300 new cases it is the ninth most common cause of cancer, and was responsible for 150,200 cancer-associated deaths in 2008 (1). Histologically, bladder urothelial carcinoma accounts for $>90 \%$ of newly diagnosed bladder malignancies $(2,3)$. However, the cause of bladder cancer, like the majority of other types of human cancer, remains to be defined, although tobacco smoking is linked to $\sim 50 \%$ of bladder cases (4). Clinically, early symptoms of bladder cancer include macroscopic and/or microscopic blood in the urine, pain during urination and frequent urination, all of which are non-specific (5). The early stage of bladder cancer recurs in 50-70\% of patients. Seeing as advanced stages of the disease remain difficult to control, effective therapeutic control of tumor recurrence is required at an early stage (5). Thus, further studies of bladder cancer are required to better understand the molecular mechanisms underlying urothelial carcinogenesis in order to provide novel strategies to prevent bladder carcinogenesis, diagnose bladder cancer early and effectively treat or cure bladder cancer in clinic.

Cell adhesion molecule 1 (CADM1) is a novel tumor suppressor gene that is localized at chromosome 11q23.2. CADM1 protein is a transmembrane glycoprotein with 442 amino acids and belongs to an immunoglobulin cell adhesion superfamily $(6,7)$; thus, CADM1 protein has structural homology to the neural cell adhesion molecules and cell signaling transduction (8) and is implicated in calcium ion independent cell-cell adhesion (9). CADM1 was reported to be abrogated or significantly reduced in a number of human cancer tissues and cell lines, including esophageal cancer (10), cutaneous melanoma (11), hepatocellular carcinoma (12), ovarian carcinoma (13), breast cancer $(14,15)$, pancreatic ductal adenocarcinoma (16), lung cancer (17), laryngeal squamous cell carcinoma (18), colorectal cancer (19), prostate cancer (20), neuroblastoma (21) and nasopharyngeal carcinoma $(22,23)$. Lost CADM1 expression was associated with poor prognosis and development of esophageal cancer (10) and ovarian cancer (13). CADM1 promoter hypermethylation may be responsible for lost CADM1 expression $(16,19,20)$. In bladder cancer, numerous previous studies analyzed altered 
methylation of $C A D M 1$ promoter in non-muscle-invasive bladder cancer (24-26) and bladder cancer with various stages (27). The results revealed an increase in methylation of $C A D M 1$ promoter in non-muscle-invasive bladder cancer, with the exception of the study by Hellwinkel et al (27). To assess the contradiction, the present study further investigated the expression and the function of CADM1 in bladder tissues. In order to assess the associations with clinicopathological data of patients, we detected the methylation status of $\mathrm{GpG}$ islands of CADM1 promoter and protein expression levels of bladder cancer tissues. Then we investigated the effects of CADM1 overexpression on the regulation of bladder cancer cell viability, apoptosis, invasion and gene expression in vitro. The present study is expected to provide useful evidence for CADM1 as a biomarker for early detection of bladder cancer and prediction of disease progression and it may be a novel target for future control of bladder cancer in clinical practice.

\section{Materials and methods}

Tissue specimens. A total of 84 bladder cancer tissues from patients with bladder cancer (58 male with mean age of $68.5 \pm 9.9$ years and 26 female with mean age of $69.4 \pm 7.3$ years) and 20 normal bladder mucosae were collected from patients with benign prostate hyperplasia were collected from the Department of Urology, The Second Hospital of Tianjin Medical University (Tianjin, China) between March 2012 and April 2014. All tissue specimens were collected within 30 min following surgery, snap-frozen and stored in an $-80^{\circ} \mathrm{C}$ freezer. Bladder cancer was histopathologically confirmed by Jiwu Chang and Aixiang Wang, pathologists of The Tianjin Institute of Urology, according to the 2004 WHO classification of tumor of urinary system (28) and 2009 TNM classification of malignant tumors (29). The present study also obtained $2 \mathrm{ml}$ peripheral blood samples from each of the 20 cancer-free individuals. The present study was approved by the Ethics Committee of The Second Hospital of Tianjin Medical University and written informed consent was obtained from each patient prior to enrollment in the present study.

Cell line and culture. The human bladder cancer T24 cell line was obtained from Tianjin Institute of Urology (Tianjin, China) and cultured in Dulbecco's modified Eagle's medium (DMEM) supplemented with $10 \%$ fetal bovine serum (Invitrogen; Thermo Fisher Scientific, Inc., Waltham, MA, USA), penicillin (100 U/ml; Sigma-Aldrich; Merck KGaA, Darmstadt, Germany) and streptomycin (100 $\cdot \mathrm{g} / \mathrm{ml}$; Sigma-Aldrich; Merck $\mathrm{KGaA}$ ) in a humidified incubator with $5 \% \mathrm{CO}_{2}$ at $37^{\circ} \mathrm{C}$.

Bisulfite modification and methylation specific PCR. Genomic DNA was extracted from bladder cancer tissues, normal bladder mucosae and peripheral blood mononuclear cells using the E.Z.N.A. ${ }^{\text {TM }}$ Tissue DNA kit (Omega Bio-Tek, Norcross, GA, USA). The purity and concentration of DNA was measured using a DU800 ultraviolet spectrophotometer (Beckman-Coulter, Inc., Brea, CA, USA). Following quantification, these DNA samples were subjected to bisulfite modification using EZ DNA Methylation-Gold kit TM (Omega Bio-Tek). Briefly, $1 \mu \mathrm{g}$ genomic DNA sample of each was incubated with the kit reagents, according to the manufacturer's protocol, and $4 \mu 1$ of the treated DNA samples were amplified by PCR using primers specific for either the unmethylated or methylated template in a $50 \mu \mathrm{l}$ PCR mixture. Primers used for this methylated reaction were as follows: Forward, 5'-TTT TAATTATTATTTTCGAGTTTATCG-3' and reverse, 5'-TCT TTAAAAACGAAAACTATACG-3', and primers for the unmethylated reaction were: Forward, 5'-TTTTTTAATTAT TTTTGAGTTTATTG-3' and reverse, 5'-AAATCTTTAAAA ACAAAAACTATAC-3'. PCR amplification conditions were set for an initial denaturation at $95^{\circ} \mathrm{C}$ for $10 \mathrm{~min}$, followed by 35 cycles of $94^{\circ} \mathrm{C}$ for $30 \mathrm{sec}, 57^{\circ} \mathrm{C}$ (for methylated primers) or $55^{\circ} \mathrm{C}$ (for unmethylated primers) for $30 \mathrm{sec}$ and a final extension at $72^{\circ} \mathrm{C}$ for $3 \mathrm{~min}$. Genomic DNA from normal pancreatic tissues treated with or without SssI methyltransferase was used as a positive or negative control, respectively. The PCR products were then separated in $2 \%$ agarose gels, stained with ethidium bromide and visualized under UV illumination.

Construction of lentiviral vector carrying CADM1 cDNA and short interfering (si)RNAs, production of viral particles and viral infection. Human CADM1 full-length cDNA (accession number NM_014333) was amplified using reverse transcription (RT)-PCR with CADM1-specific primers containing XhoI and NotI restriction enzyme sites (forward, 5'-AAT CTCGAGATGGCGAGTGTAGTGCTGCC-3' and reverse, 5'-ATAGCGGCCGCCTAGATGAAGTACTCTTTCT-3').

PCR was performed using the LA Taq ${ }^{\mathrm{TM}}$ System (Takara Biotechnology Co., Ltd., Dalian, China) for 30 cycles of $94^{\circ} \mathrm{C}$ for $40 \mathrm{sec}, 55^{\circ} \mathrm{C}$ for $60 \mathrm{sec}$ and $72^{\circ} \mathrm{C}$ for $50 \mathrm{sec}$, according to the manufacturer's protocol. The PCR products were ligated into the pHBLV-IRES-ZsGreen-PGK-puro vector (Hanbio Biotechnology Co., Ltd., Shanghai, China) to obtain the Ad-CADM1 vector. Furthermore, 3 siRNAs were also designed to silence CADM1 expression and the DNA sequences of these siRNAs were: siRNA1, 5'-CAGATGACTTATCCTCTA CAA-3'; siRNA2, 5'-CCAGACATAAAGGTACATACT-3'; and siRNA3, 5'-AGACGCAGACACAGCTATAAT-3'. These DNA sequences were ligated into the pHBLV-U6-ZsGreen-Puro vector (Hanbio Biotechnology Co., Ltd.) to obtain sh-CADM1. These lentivirus pHBLV-IRES-ZsGreen-PGK-puroCADM1 (Ad-CADM1), pHBLV-U6-ZsGreen-Puro-sh1/2/3 (Ad-sh1/2/3) or pHBLV-U6-ZsGreen-Puro (Ad-GFP2), and lentivirus packaging plasmid pSPAX2 and pMD2G or pHBLV-IRES-ZsGreen-PGK-puro (Ad-GFP1) were then using Lipofectamine $^{\mathrm{TM}} 2000$ (Life Technologies) according to the manufacturer's instructions into human embryonic kidney 293T cells for homologous recombination and production of lentiviral particles. Each recombinant virus was purified by ultracentrifugation at $4^{\circ} \mathrm{C}$ and at $72,000 \mathrm{x}$ g for $120 \mathrm{~min}$. Titers of these viral particles were assessed for multiplicity of infection (MOI).

Human bladder cancer T24 cells were plated at a density $5 \times 10^{5}$ cell in 6-wells plates and grown overnight prior to infection with these lentiviruses at an MOI of 20 for $24 \mathrm{~h}$ at $36^{\circ} \mathrm{C}, 48$ and $72 \mathrm{~h}$ at $36^{\circ} \mathrm{C}$. Expression of green fluorescence protein (GFP) was detected under a fluorescence microscope for infection efficiency in $\geq 5$ fields of view.

$R T-q P C R$. Total RNA was isolated from parental T24, T24-Ad-CADM1, T24-Ad-sh1/2/3 and T24-Ad-GFP1/2 cells using the TRIzol reagent (Invitrogen; Thermo Fisher Scientific, 
Inc.), according to the manufacturer's protocol. qPCR was performed on ABI 790096 HT series PCR machine (Applied Biosystems; Thermo Fisher Scientific, Inc.) using FastStart Universal SYBR Green Master (ROX) kit (Roche). qPCR was then performed with the specific CADM1 primers (forward, 5'-ATGGCGAGTGTAGTGCTGC-3' and reverse, 5'-GAT CACTGTCACGTCTTTCGT-3'). Relative CADM1 expression level was then normalized to levels of GAPDH (forward, 5'-GGAGCGAGATCCCTCCAAAAT-3' and reverse, 5'-GGC TGTTGTCATACTTCTCATGG-3') as an internal control. PCR conditions were $50^{\circ} \mathrm{C}$ for $2 \mathrm{~min}$ and $95^{\circ} \mathrm{C}$ for $10 \mathrm{~min}$ followed by 40 cycles of $95^{\circ} \mathrm{C}$ for $15 \mathrm{sec}$, and $60^{\circ} \mathrm{C}$ for $1 \mathrm{~min}$. The expression levels were normalized against glyceraldehyde 3-phosphatedehydrogenase (GAPDH) (Human GAPDH Endogenous Control, Applied Biosystems; Thermo Fisher Scientific, Inc.). The results were summarized from triplicate experiments using the $2^{-\Delta \Delta C q}$ method (30).

Protein extraction and western blotting. Total cellular protein was extracted from parental T24, T24-Ad-CADM1, T24-Ad-sh1/2/3 and T24-Ad-GFP1/2 cells or from tissue specimens using a lysis buffer containing PBS, Triton X-100, PMSF, leupeptine and pepstain (Boster Biological Technology, Pleasanton, CA, USA). Following quantification using a Bio-Rad Protein Assay kit II (Bio-Rad Laboratories, Inc., Hercules, CA, USA), these protein samples (15 $\mu \mathrm{g}$ of each tissue or cell lysate) were subjected to 10-15\% SDS-PAGE and transferred onto polyvinylidene fluoride membranes (EMD Millipore, Billerica, MA, USA). The Western blotting was performed according to standard methods using antibodies against CADM1 (1:1,000 dilution; catalog no. H00023705-M02; Abnova, Taipei, Taiwan) and GAPDH (1:1,000 dilution; catalog no. ab70699), Caspase-3 (1:1,000 dilution; catalog no. ab2171), Bcl-2 (1:1,000 dilution; catalog no. ab32124), Bax (1:1,000 dilution; catalog no. ab32503E-cadherin (1:1,000 dilution; catalog no. ab1416), $\beta$-catenin (1:1,000 dilution; catalog no. ab16051), vimentin (1:1,000 dilution; catalog no. ab92547), p27 (1:1,000 dilution; catalog no. ab32034), cyclinD1 (1:1,000 dilution; catalog no. ab134175), cyclinE1 (1:1,000 dilution; catalog no. ab3927), CDK2 (1:1,000 dilution; catalog no. ab32147) (all Abcam, Cambridge, UK). Briefly, the membranes were blocked with $5 \%$ skimmed milk solution in PBS for $1 \mathrm{~h}$ at the room temperature and incubated with a rat anti-CADM1, followed by biotinylated goat anti rat IgG secondary antibody (1:1,000 dilution; catalog no. SAB4600463; Sigma-Aldrich; Merck KGaA) for $2 \mathrm{~h}$ at $37^{\circ} \mathrm{C}$. The membranes were incubated with enhanced chemiluminescence solution (PerkinElmer, Inc., Waltham, MA, USA). The images were acquired using an Image Quant350 digital image system (GE Healthcare Life Sciences, Chalfont, UK). Expression levels of proteins were semi-quantified by evaluating the gray scale using Image-ProPlus version 5.0 software (Media Cybernetics, Inc., Rockville, MD, USA). Expression levels of GAPDH protein were used as a loading control. The relative expression levels of protein were determined as the CADM1/GAPDH ratio.

Cell viability assay. Cellular viability was assessed using an MTT assay, according to the manufacturer's protocol. Briefly, parental T24, T24-Ad-CADM1, T24-Ad-sh1 and T24-Ad-GFP1/2 cells were cultured for 24, 48, 72 and $96 \mathrm{~h}$ at $37^{\circ} \mathrm{C}$. The cells were further incubated with $0.5 \mathrm{mg} / \mathrm{ml} \mathrm{MTT}$ for $4 \mathrm{~h}$ at $37^{\circ} \mathrm{C}$ and the culture medium was replaced with $200 \mu \mathrm{l}$ dimethyl sulfoxide to dissolve the purple precipitates for $20 \mathrm{~min}$ at room temperature. The absorbance rate was evaluated at $490 \mathrm{~nm}$ using a multi-well plate reader. The experiments were performed in triplicate and repeated $\geq 3$ times.

Tumor cell invasion assay. Cell invasion capacity was assayed using 24-well Transwell chambers ( $8 \mathrm{~mm}$ pore size; BD Biosciences, Franklin Lakes, NJ, USA), according to the manufacturer's protocol. In brief, $150 \mu 12 \times 10^{5} / \mathrm{ml}$ parental T24, T24-Ad-CADM1, T24-Ad-sh1 and T24-Ad-GFP1/2 cells in serum-free medium were seeded into the upper chambers, and $800 \mu 1$ DMEM supplemented with $10 \%$ fetal bovine serum was added to the lower chambers and cultured for $24 \mathrm{~h}$ at $37^{\circ} \mathrm{C}$. Cells that remained in the upper chambers were removed with a cotton swab and the cells that invaded through the Matrigel and attached to the lower surface of the membrane were fixed and stained with $1 \%$ crystal violet solution. Subsequently, the membrane of each chamber was removed, mounted on microslides and was fixed using permount ${ }^{\mathrm{TM}}$ mounting medium (Electron Microscopy Sciences, Hatfield, PA, USA). In total, 6 random fields were used to capture images under a light microscope and counted. Each cell line preparation was seeded into 4 chambers and the cell density in each chamber was $2 \times 10^{5}$ cells $/ \mathrm{ml}$.

Flow cytometric cell cycle assay. Parental T24, T24-AdCADM1, T24-Ad-sh1 and T24-Ad-GFP1/2 cells were harvested and single-cell suspensions were prepared by passing the cells through a nylon mesh. Subsequently, cells were fixed in $70 \%$ ethanol at $-20^{\circ} \mathrm{C}$ for $2 \mathrm{~h}$, washed 3 times with ice-cold PBS and suspended in propidium iodide (PI) buffer (Invitrogen; Thermo Fisher Scientific, Inc.) with cells adjusted to a density of $4 \times 10^{6} / \mathrm{ml}$. The cells were then incubated with $5 \mu \mathrm{g} / \mathrm{ml}$ propidium iodide (Sigma-Aldrich; Merck KGaA) at room temperature for $30 \mathrm{~min}$ in the dark. The single cells were then subjected to FACScan analysis (Becton-Dickinson, Heidelberg, Germany). Cell cycle distribution was calculated using ModFIT cell cycle analysis software (version 2.01.2; Becton Dickinson). Each experiment was repeated at least three times on different days for validation.

Statistical analysis. All statistical analyses were performed using SPSS version 17.0 (SPSS Inc., Chicago, IL, USA). The data are expressed as the mean \pm standard deviation, except when otherwise stated. The Mann-Whitney-U test was performed to compare protein concentrations in tissue specimens. $\chi^{2}$ test was used to compared the values of the test and control samples statistically. $\mathrm{P}<0.05$ was considered to indicate a statistically significant difference.

\section{Results}

Association of CADM1 promoter methylation with bladder cancer progression ex vivo. In the present study, methylation of status CADM1 promoter was assessed in 84 bladder cancer tissues vs. 20 normal bladder mucosae and it was revealed that CADM1 promoter was methylated in 43/84 (51.2\%) bladder urothelium carcinoma tissues vs. none of the 20 normal 
Table I. Association of CADM1 promoter methylation with clinicopathological features from bladder cancer patients.

\begin{tabular}{|c|c|c|c|c|c|}
\hline Clinicopathological features & Number & Methylated, n (\%) & Unmethylated, n (\%) & $\chi^{2}$ value & P-value \\
\hline \multicolumn{6}{|l|}{ Gender } \\
\hline Male & 58 & $34(58.6)$ & $24(41.4)$ & \multirow[t]{2}{*}{1.12} & \multirow[t]{2}{*}{0.346} \\
\hline Female & 26 & $14(53.8)$ & $12(46.2)$ & & \\
\hline \multicolumn{6}{|l|}{ Age, years } \\
\hline$\leq 65$ & 33 & $18(54.5)$ & $15(45.5)$ & \multirow[t]{2}{*}{0.044} & \multirow[t]{2}{*}{1} \\
\hline$>65$ & 51 & $29(56.9)$ & $22(43.1)$ & & \\
\hline \multicolumn{6}{|l|}{ Tumor number } \\
\hline Single & 34 & $20(58.8)$ & $14(41.2)$ & \multirow[t]{2}{*}{0.23} & \multirow[t]{2}{*}{0.654} \\
\hline Multiple & 50 & $32(64.0)$ & $18(36.0)$ & & \\
\hline \multicolumn{6}{|l|}{ Tumor recurrent } \\
\hline Yes & 49 & $33(67.3)$ & $16(32.7)$ & \multirow[t]{2}{*}{7.51} & \multirow[t]{2}{*}{0.008} \\
\hline No & 35 & $13(37.1)$ & $22(62.9)$ & & \\
\hline \multicolumn{6}{|l|}{ Tumor size $(\mathrm{cm})$} \\
\hline$\leq 3$ & 49 & $21(42.9)$ & $28(57.1)$ & \multirow[t]{2}{*}{5.42} & \multirow[t]{2}{*}{0.027} \\
\hline$>3$ & 35 & $24(68.6)$ & $11(31.4)$ & & \\
\hline \multicolumn{6}{|l|}{ Tumor grade } \\
\hline Low & 57 & $25(43.9)$ & $32(56.1)$ & \multirow[t]{2}{*}{6.72} & \multirow[t]{2}{*}{0.011} \\
\hline High & 27 & $20(74.1)$ & $7(25.9)$ & & \\
\hline \multicolumn{6}{|l|}{ TNM stage } \\
\hline Ta- $\mathrm{T}_{1}$ & 50 & $22(44.0)$ & $28(56.0)$ & \multirow[t]{2}{*}{8.71} & \multirow[t]{2}{*}{0.004} \\
\hline $\mathrm{T}_{2}-\mathrm{T}_{4}$ & 34 & $26(76.5)$ & $8(23.5)$ & & \\
\hline
\end{tabular}

CADM1, cell adhesion molecule 1; TNM, tumor-node-metastasis.

A

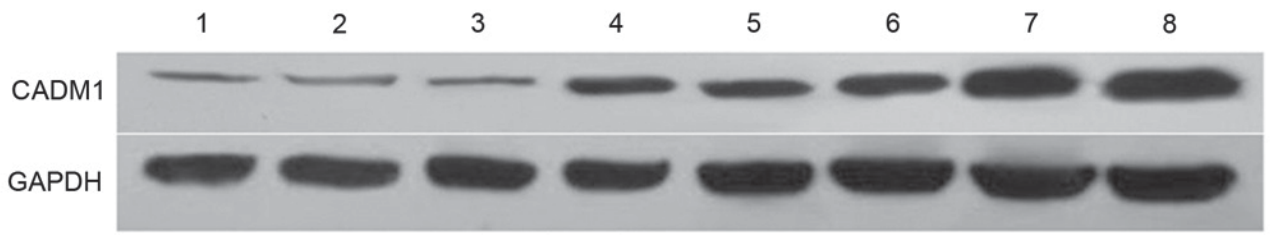

B Marker $\frac{\text { Case } 1}{\mathrm{MU}} \frac{\text { Case } 2}{\mathrm{MU}} \frac{\text { Case } 3}{\mathrm{MU}} \frac{\text { Case } 4}{\mathrm{MU}} \frac{\text { Case } 5}{\mathrm{MU}} \frac{\text { Case } 6}{\mathrm{MU}} \frac{\text { Case } 7}{\mathrm{MU}} \frac{\text { Case } 8}{\mathrm{MU}} \frac{\text { Positive }}{\mathrm{MU}} \frac{\text { Negative }}{\mathrm{MU}}$

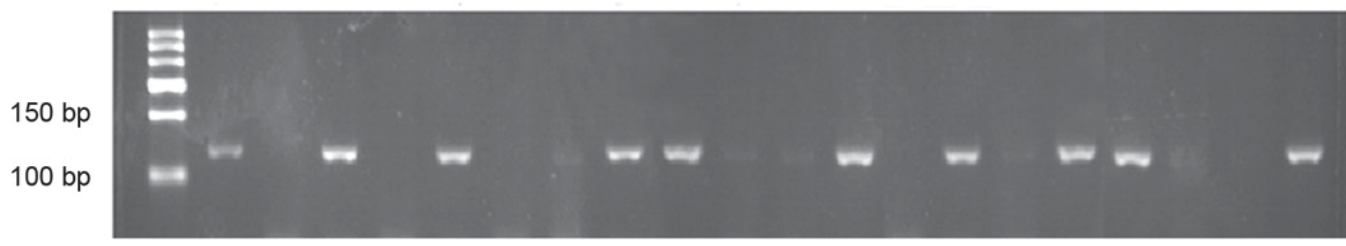

Figure 1. Downregulation of CADM1 expression and methylation of CADM1 promoter in bladder cancer tissues. (A) Western blot analysis of the expression level of CADM1 protein bladder cancer vs. normal tissues. Lanes 1-6 are tumor tissues and lanes 7-8 are normal bladder tissues. (B) The methylation of CADM1 promoter was assayed using methylation specific PCR in bladder tissue samples. CADM1, cell adhesion molecule 1.

tissues (Table I). The methylation status of the CADM1 promoter was significantly different between normal bladder mucosae and bladder cancer tissues $(\mathrm{P}<0.01$; Table I). The methylation of CADM1 promoter in bladder cancer tissues was significantly associated with tumor size, recurrence, pathology classification and clinical stage $(\mathrm{P}<0.05$; Table I), but not with patients age, gender and tumor number $(\mathrm{P}>0.05)$.

Association of CADM1 promoter methylation with expression level of CADM1 protein in bladder tissues. The present study 

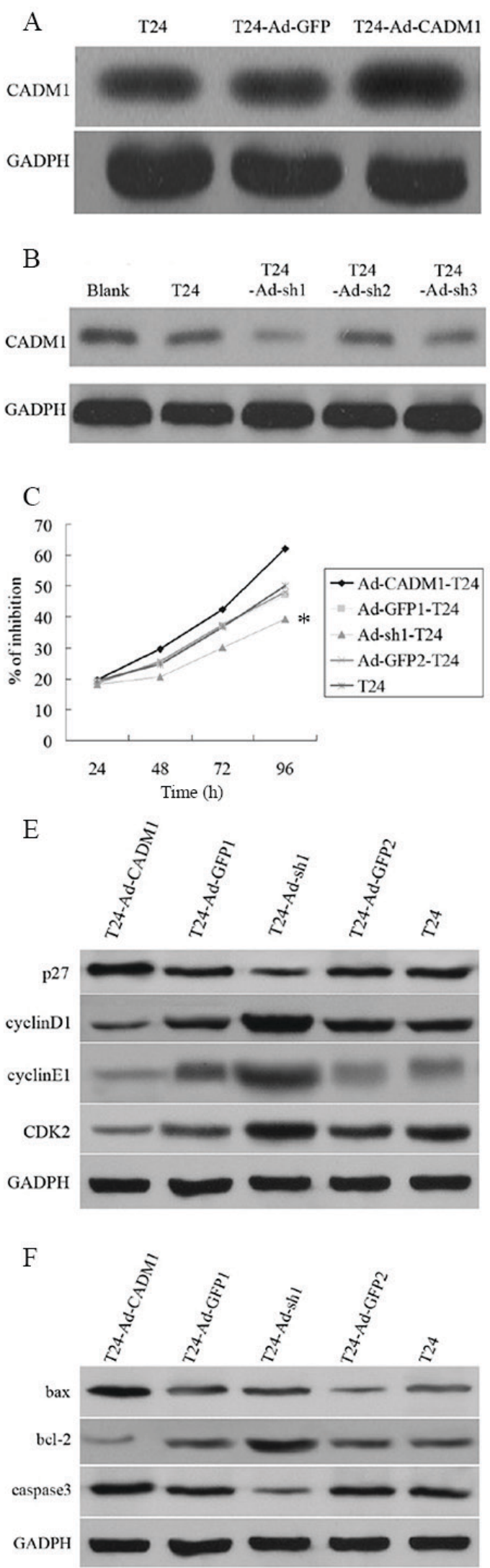
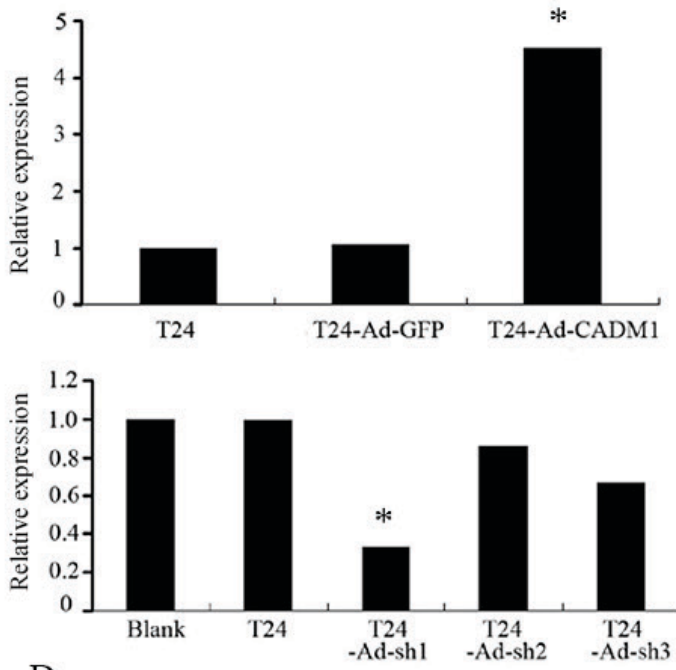

$\mathrm{D}$
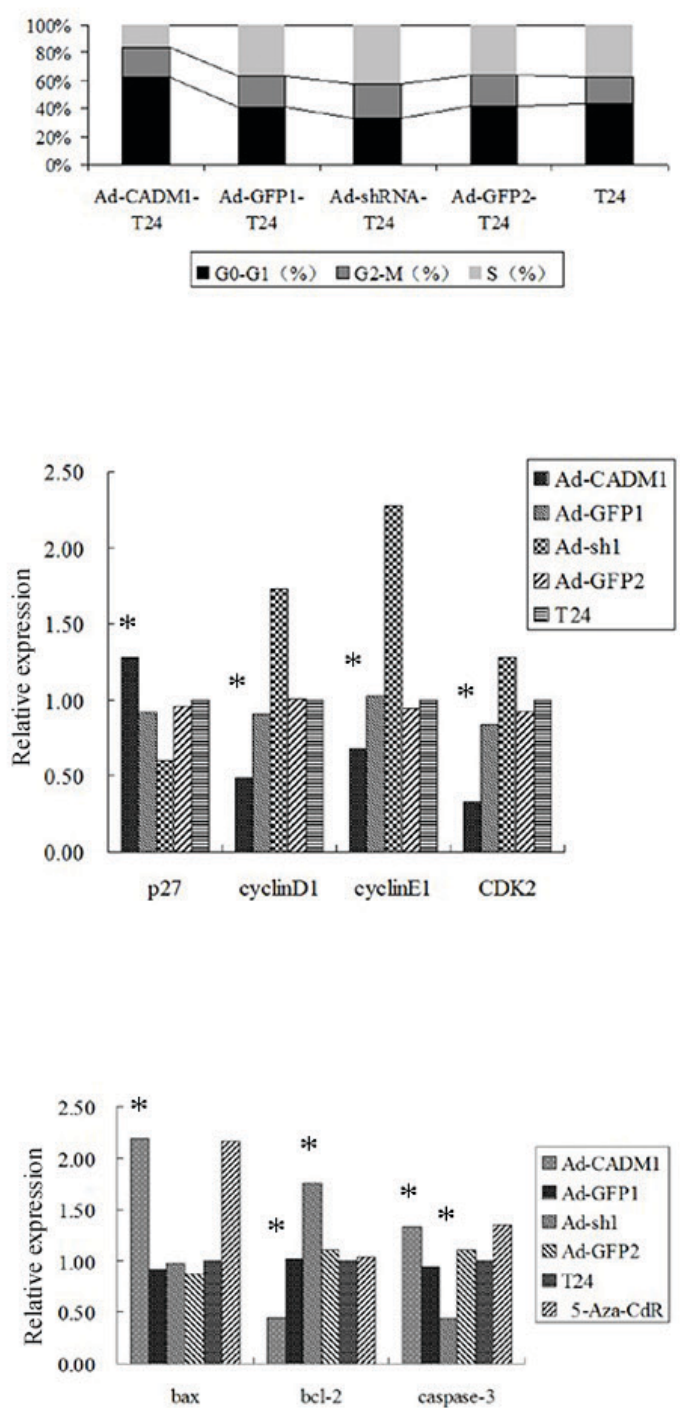

Figure 2. Effects of CADM1 overexpression or knockdown in regulation of bladder cancer cell viability and cell cycle progression. (A) Western blot analysis of T24 bladder cancer cells infected with lentivirus carrying CADM1 cDNA for overexpression of CADM1 protein. The CADM1 protein was highest in T24-Ad-CADM1 group "P<0.05. (B) Western blot analysis of T24 bladder cancer cells infected with lentivirus carrying CADM1 siRNAs to knockdown expression of CADM1 protein. The CADM1 protein exhibited reduced expression the in T24-Ad-sh1 group "P<0.05. (C) MTT assay to determine cell viability of lentivirus infected T24 bladder cancer cells. The most effective on inhibition of cell viability was identified in the Ad-sh1-T24 group "P<0.05. (D) Flow cytometric cell cycle assay. The duplicated cells were subjected to flow cytometric assay. Western blot analysis to determine expressions levels of (E) p27, cyclinD1, cyclinE1, CDK2 and GAPDH and (F) bax, bcl-2, caspase 3 and GAPDH. "P<0.05. CADM1, cell adhesion molecule 1; CDK2, cyclin dependent kinase 2; bcl-2, B cell lymphoma 2; sh, short hairpin. 

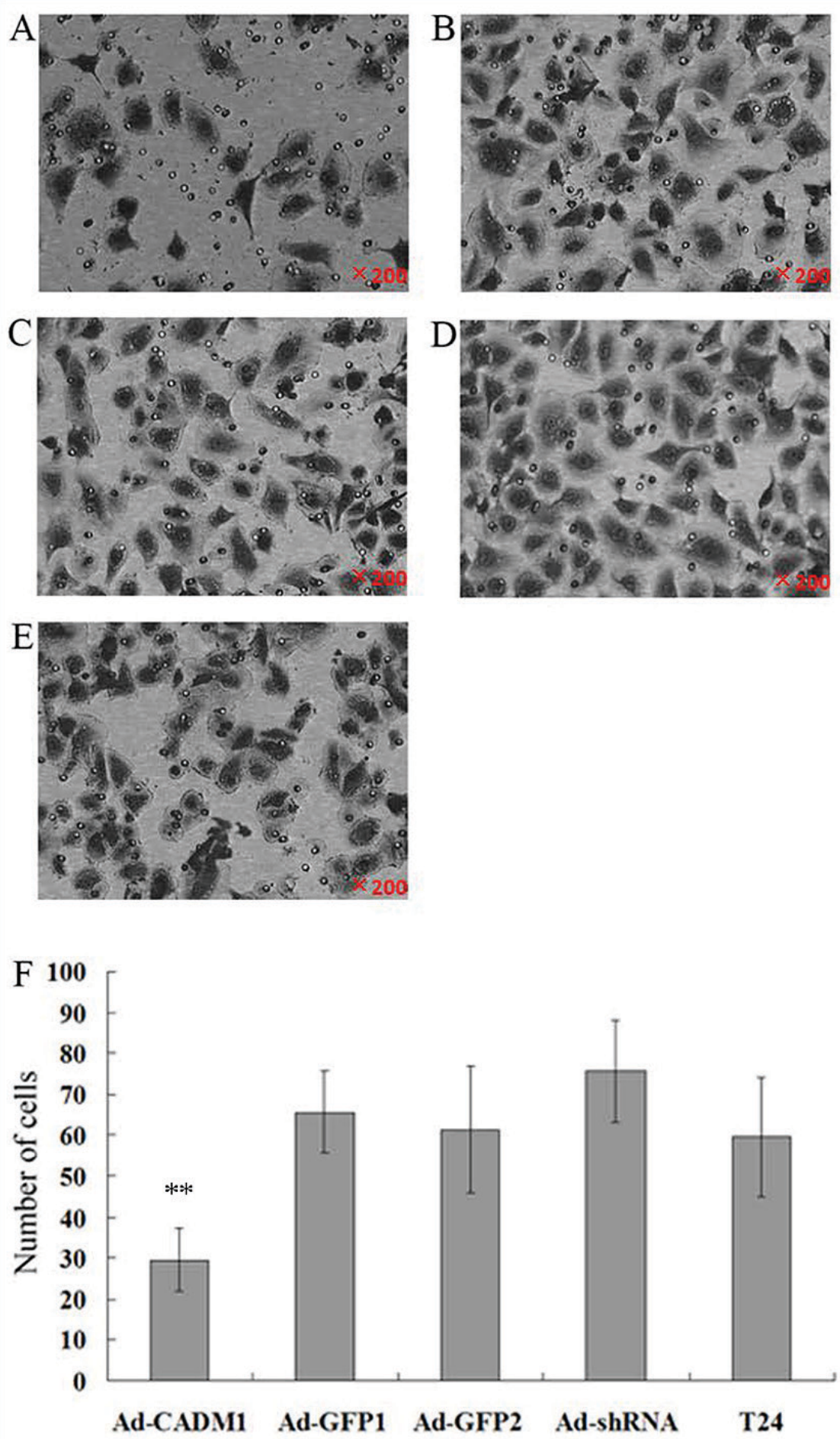

Figure 3. Effects of CADM1 overexpression or knockdown on regulation of bladder cancer cell invasion capacity. T24 bladder cancer cells were infected with lentivirus carrying CADM1 cDNA or siRNA and then subjected to Transwell tumor cell invasion assay (x200 magnification). (A) T24-Ad-CADM1. (B) T24-Ad-GFP1. (C) T24-Ad-shRNA. (D) T24-Ad-GFP2. (E) T24. (F) The numbers of cells invaded into the lower chamber of various cell groups. Decreased invasion was identified following overexpression of CADM1 in the Ad-CADM1 group " $\mathrm{P}<0.01$. CADM1, cell adhesion molecule 1; shRNA, short hairpin RNA; siRNA, short interfering RNA.

analyzed levels of CADM1 protein in fresh bladder tissues and demonstrated that CADM1 protein expression level was lower in bladder cancer tissues compared with in normal tissues (0.261 \pm 0.141 vs. $0.696 \pm 0.092 ; \mathrm{P}<0.01$; Fig. 1$)$.

Effects of CADM1 overexpression and knockdown on the regulation of bladder cancer cell viability, apoptosis and cell cycle distribution. The biological effects of CADM1 on bladder cancer were investigated using infected T24 bladder cancer cells with lentivirus carrying CADM1 cDNA or siRNAs to established CADM1 stable overexpression cell line T24-Ad-CADM1 and or knockdown cell line T24-Ad-sh1/2/3, compared with the empty vector-transfected control cell line T24-Ad-GFP1/2. The results revealed that 

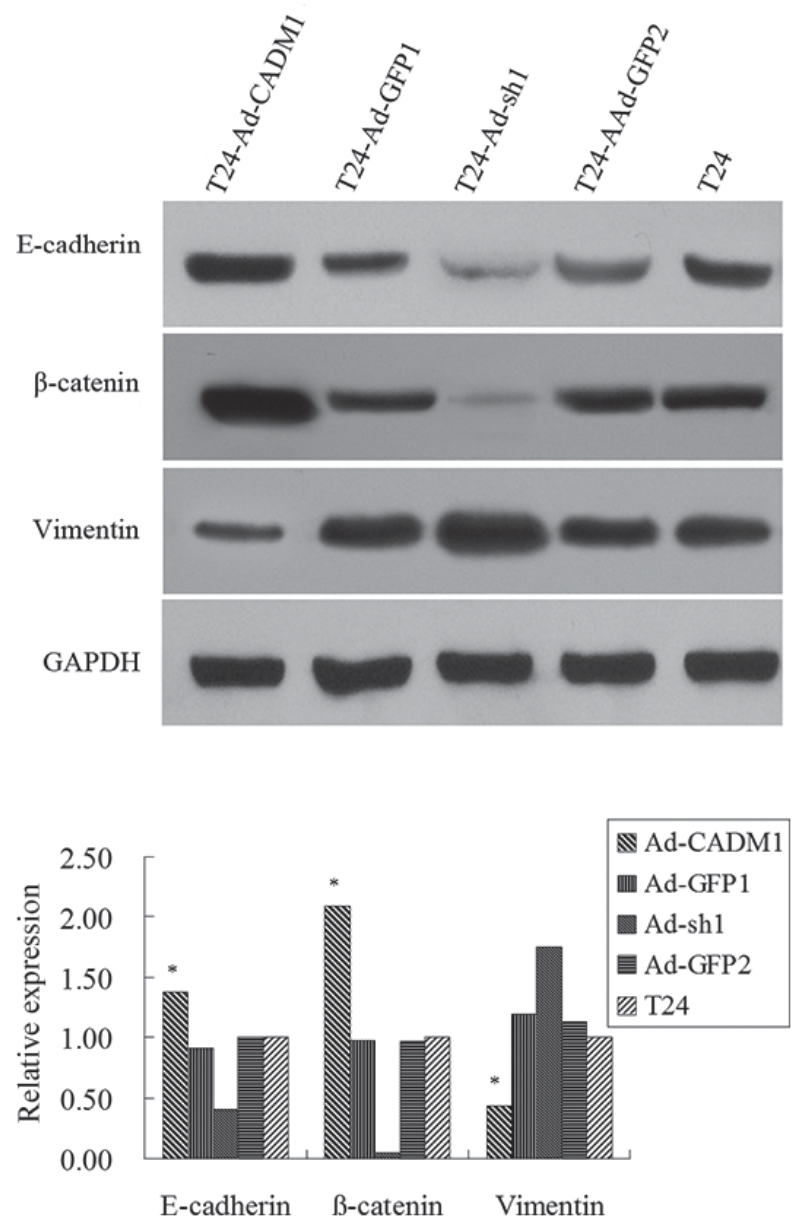

Figure 4. Effects of CADM1 overexpression or knockdown on the regulation of tumor cell EMT. T24 bladder cancer cells were infected with lentivirus carrying CADM1 cDNA or siRNA and then subjected to western blot analysis of EMT-associated gene expression levels. EMT, epithelium to mesenchymal transition; CADM1, cell adhesion molecule 1; siRNA, short interfering RNA. "P $<0.05$.

lentivirus carrying CADM1 cDNA overexpressed CADM1 protein, whereas lentivirus carrying CADM1 siRNA reduced CADM1 expression level in T24 bladder cancer cells (Fig. 2A and B).

The present study then assessed the altered phenotypes of the T24 bladder cancer cells using MTT and cell cycle flow cytometric assays and demonstrated that overexpression of CADM1 reduced cell viability and arrested cells in the $\mathrm{G}_{1} / \mathrm{G}_{0}$ phases of the cell cycle (Fig. $2 \mathrm{C}$ and D). However, knockdown of CADM1 expression level promoted cell growth and cell cycle progression to the $\mathrm{S}$ phase (Fig. 2D).

Effects of CADM1 overexpression and knockdown on the regulation of gene expression in T24 bladder cancer cells. To further explore the molecular mechanisms underlying CADM1 overexpression inhibition of T24 cell proliferation, expression levels of cell growth and cell cycle-associated proteins, including p27, cyclinD1, CDK2 and cyclinE1, were determined. It was revealed that the expression level of p27 protein in T24-Ad-CADM1 cells was significantly higher compared with in parental T24 cells and T24-Ad-GFP1 cells $(\mathrm{P}<0.05)$. Whereas, the expression levels of cyclinD1, CDK2, and cyclinE1 protein in T24-Ad-CADM1 cells was markedly downregulated compared with in parental T24 cells and T24-Ad-GFP1 cells (P<0.05; Fig. 2E). Conversely, CADM1 knockdown had a contrary effect (Fig. 2E). Furthermore, the present study also assessed the altered apoptosis-associated gene expression level and the data demonstrated that the expression levels of caspase- 3 and Bax proteins were increased and bcl- 2 protein was markedly decreased in T24-Ad-CADM1 cells compared with in parental T24 cells and T24-Ad-GFP1 ( $\mathrm{P}<0.05$; Fig. 2F). Conversely, the expression level of caspase-3 protein was markedly decreased and bcl-2 protein was increased in T24-Ad-sh1 cells compared with in parental T24 cells and T24-Ad-GFP2 (P<0.05; Fig. 2F).

Effects of CADMI overexpression on regulation of tumor cell invasion. Since CADM1 functions to regulate cell adhesion and mobility, the present study assessed CADM1 overexpression or knockdown in the regulation of tumor cell invasion and gene expression. The tumor cell invasion results revealed that the numbers of cells invaded into the lower chamber in T24-Ad-CADM1, T24-Ad-GFP1, T24-Ad-sh1, T24-Ad-GFP2 and parental T24 cells were $29.54 \pm 7.66,65.57 \pm 10.02,75.61 \pm 12.53,61.32 \pm 15.43$ and $59.54 \pm 14.58$, respectively. Thus, compared with in parental T24 and T24-Ad-GFP1 cells, the overexpression of CADM1 significantly suppressed tumor cell invasion capacity $(\mathrm{P}<0.01$; Fig. 3). However, CADM1 knockdown significantly increased tumor cell invasion $(\mathrm{P}<0.01$; Fig. 3$)$.

Furthermore, the present study assessed the expression levels of key factors or markers of epithelial to mesenchymal transition (EMT), including E-cadherin, $\beta$-catenin and Vimentin. The results revealed that the expression levels of E-cadherin and $\beta$-catenin proteins were markedly increased in T24-Ad-CADM1 cells compared with those in parental T24 and T24-Ad-GFP1 cells, whereas the expression level of Vimentin protein was decreased in T24-Ad-CADM1 cells compared with in parental T24 and T24-Ad-GFP1 cells $(\mathrm{P}<0.05$; Fig. 4). Conversely, CADM1 knockdown had the opposite effects on the expression level of these proteins (Fig. 4). These results illustrated that the upregulation of CADM1 expression level was able to inhibit EMT in T24 cells.

\section{Discussion}

The present study first assessed methylation of CADM1 promoter and CADM1 protein expression level in bladder tissues for associations with clinicopathological parameters from bladder cancer patients. Subsequently, the effects of CADM1 overexpression and knockdown on the regulation of bladder cancer cell viability, cell cycle distribution, apoptosis, invasion capacity in vitro and the underlying molecular events, were investigated. The results demonstrated that the CADM1 promoter was highly methylated in bladder cancer tissues compared with in normal mucosae tissues, which induced a reduced CADM1 protein expression level in bladder cancer tissues. Methylated CADM1 promoter was significantly associated with tumor size, recurrence, pathology classification and advanced clinical stages. Furthermore, overexpression of CADM1 protein inhibited tumor cell proliferation, reduced cell invasion and induced cell apoptosis, whereas knockdown 
of CADM1 expression promoted tumor cell growth, invasion capacity and cell cycle progression. At the gene level, overexpression of CADM1 protein increased the expression levels of caspase-3, Bax and p27 proteins, but decreased the expression levels of bcl-2, cyclinD1, cyclinE1 and CDK2 proteins. CADM1 knockdown had an opposite effect on the expression level of these proteins. Overexpression of CADM1 protein regulated the expression level of EMT markers, including increased expression levels of E-cadherin and $\beta$-catenin, whereas it decreased the expression levels of Vimentin. Thus, the present study demonstrated that reduced CADM1 expression levels may contribute to bladder cancer development and progression and targeting CADM1 expression may be used as a novel therapeutic strategy in the future for controlling bladder cancer.

The present study determined methylation status of the CADM1 promoter for association with clinicopathological parameters. The results demonstrated that CADM1 promoter methylation induced lost CADM1 expression in bladder cancer tissues, which was also associated with tumor progression ex vivo. Previous studies revealed that methylation of CADM1 promoter occurred in non-muscle-invasive bladder cancer (24-26) and the present study confirmed that methylation of CADM1 promoter also increased in Ta and T1 stage bladder cancer; however, these results were contrary to those by Hellwinkel et al (27) demonstrating that methylation of CADM1 promoter was not significant in bladder cancer with various stages: Their sample size was small but there were more advanced stage bladder cancers. Of note, increasing evidence has revealed that CADM1 is a critical regulator of cell proliferation, invasion and apoptosis $(12,17-19,31,32)$. For example, Mao et al (31) revealed that overexpression of CADM1 inhibited lung cancer cell proliferation, induced tumor cell apoptosis and increased caspase-3 activity. It was further demonstrated that compared with the control group, administration of CADM1 suppressed tumor growth in nude mice to the extent of 70-80\% (31). Lu et al (18) reveled that expression levels of CADM1 mRNA and protein was significantly reduced in laryngeal squamous cell carcinoma tissues, which was significantly associated with advanced tumor-node-metastasis staging and lymph node metastases, but not with age, gender and tumor differentiation. They also demonstrated that elevation of CADM1 expression inhibited tumor cell proliferation, reduced tumor cell invasion and induced cell apoptosis (18). Further studies are required to investigate the underlying mechanism by which CADM1 promoter is methylated and whether it is a common event in various types of human cancer.

Furthermore, to illustrate the functions of CADM1 protein in T24 bladder cancer cells, the present study performed cell viability MTT assay following manipulated CADM1 expression using lentivirus carrying CADM1 cDNA or siRNA vs. negative control vectors. It was revealed that tumor cell proliferation was markedly inhibited in T24-Ad-CADM1 compared with parental T24 and T24-Ad-GFP1 cells. Conversely, knockdown of CADM1 expression level induced tumor cell proliferation compared with that in parental T24 and T24-Ad-GFP2 cells. The present study demonstrated that CADM1 manipulation altered gene expressions, which further confirmed the effects of CADM1 on the regulation of tumor cell viability. These results suggested that CADM1 serves a critical role in regulating cell proliferation, invasion, apoptosis and cell cycle of T24 bladder cancer cells.

In addition, it is well known that EMT serves a central role in tumor invasion and metastasis. Previous studies have demonstrated that bladder cancer progression is regulated by various genes that affect tumor EMT (33-37). The present study revealed that CADM1 overexpression upregulated the expression levels of E-cadherin and $\beta$-catenin proteins in T24-Ad-CADM1 cells compared with in parental T24 and T24-Ad-GFP1 cells. Whereas, expression of vimentin protein was decreased in T24-Ad-CADM1 cells compared with in parental T24 and T24-Ad-GFP1 cells. However, CADM1 knockdown induced an opposite effect on the expression level of these proteins. The results of the presents study demonstrated that overexpression of CADM1 inhibited bladder cancer cell EMT, whereas knockdown of CADM1 expression promoted EMT.

In conclusion, the present study revealed that CADM1 expression was reduced in bladder cancer tissues and methylation of CADM1 promoter induced, and was associated with, bladder cancer progression. Furthermore, overexpression of CADM1 protein inhibited tumor cell proliferation and decreased tumor cell invasion, but induced tumor cell apoptosis and cell cycle arrest at the $\mathrm{G}_{1} / \mathrm{G}_{0}$ phase. CADM1 expression also modulated cell growth, apoptosis and cell cycle-associated proteins as well as EMT markers. The results of the present study suggested that CADM1 may be a novel gene target for clinical control of bladder cancer progression. Further studies may focus on the CADM1-regulated signaling pathway in bladder cancer.

\section{Acknowledgements}

The abstract was presented at the conference of The 2016 Huaxia Medical Forum-Genitourinary Tumor \& The 2016 Greatwall International Translational Andrology and Urology Forum (GUT-HMF 2016 \& GITAU 2016). The title of this content as follows: 'Lost expression of cell adhesion molecule 1 (CADM1) associated with bladder cancer progression and recurrence and its overexpression inhibited tumor cell malignant behaviors' and was published as abstract no. AB060 in Transl Androl Urol. 2016 Apr; 5 (Suppl 1): AB060.'

\section{Funding}

The present study was supported in by Tianjin Municipal Science and Technology Commission, Tianjin, China (grant no. 12JCZDJC23700).

\section{Availability of data and materials}

The datasets generated and/or analyzed during this study are available from the corresponding author on reasonable request.

\section{Authors' contributions}

XQL designed the study. YGC, LL, ZJG and YJY performed the experiments. YW and YJY were responsible for clinical diagnosis and sample collection. YGC and YJY contributed to data collection and statistical analysis. YGC and LL wrote 
the manuscript. All the authors read and approved the final manuscript.

\section{Ethics approval and consent to participate}

The present study was approved and monitored by the Ethics Committee of Tianjin Medical University (Tianjin, China). All procedures performed in this study were followed in accordance with the Helsinki Declaration of 1975, as revised in 2000 (38). Written informed consent was obtained from all patients and healthy individuals prior to the study.

\section{Patient consent for publication}

Written informed consent was obtained from all individual participants included in the present study.

\section{Competing interests}

All authors declare that they have no competing interest.

\section{References}

1. Jemal A, Bray F, Center MM, Ferlay J, Ward E and Forman D: Global cancer statistics. CA Cancer J Clin 61: 69-90, 2011.

2. Siegel R, Naishadham D and Jemal A: Cancer statistics, 2012. CA Cancer J Clin 62: 10-29, 2012.

3. Pashos CL, Botteman MF, Laskin BL and Redaelli A: Bladder cancer: Epidemiology, diagnosis, and management. Cancer Pract 10: 311-322, 2002.

4. Zeegers MP, Tan FE, Dorant E and van Den Brandt PA: The impact of characteristics of cigarette smoking on urinary tract cancer risk: A meta-analysis ofepidemiologic studies. Cancer 89 630-639, 2000

5. Khadjavi A, Mannu F, Destefanis P, Sacerdote C, Battaglia A, Allasia M, Fontana D, Frea B, Polidoro S, Fiorito G, et al: Early diagnosis of bladder cancer through the detection of urinary tyrosine-phosphorylated proteins. Br J Cancer 113: 469-475, 2015.

6. Murakami Y, Nobukuni T, Tamura K, Maruyama T, Sekiya T, Arai Y, Gomyou H, Tanigami A, Ohki M, Cabin D, et al: Localization of tumor suppressor activity important in nonsmal cell lung carcinoma on chromosome 11q. Proc Natl Acad Sci USA 95: 8153-8158, 1998.

7. Kuramochi M, Fukuhara H, Nobukuni T, Kanbe T, Maruyama T, Ghosh HP, Pletcher M, Isomura M, Onizuka M, Kitamura T, et al: TSLC1 is a tumor-suppressor gene in human non-small-cell lung cancer. Nat Genet 27: 427-430, 2001.

8. Shingai T, Ikeda W, Kakunaga S, Morimoto K, Takekuni K, Itoh S, Satoh K, Takeuchi M, Imai $T$, Monden $M$ and Takai Y: Implications of nectin-like molecule2/IGSF4/RA175/SgIGSF/TSLC1/SynCAM1 in cell-cell adhesion and transmembrane protein localization in epithelial cells. J Biol Chem 278: 35421-35427, 2003.

9. Yamada D, Yoshida M, Williams YN, Fukami T, Kikuchi S, Masuda M, Maruyama T, Ohta T, Nakae D, Maekawa A, et al: Disruption of spermatogenic cell adhesion and male infertility in mice lacking TSLC1/IGSF4, animmunoglobulin superfamily cell adhesion molecule. Mol Cell Biol 26: 3610-3624, 2006.

10. Ito T, Shimada Y, Hashimoto Y, Kaganoi J, Kan T, Watanabe G, Murakami Y and Imamura M: Involvement of TSLC1 in progression of esophageal squamous cell carcinoma. Cancer Res 63: 6320-6326, 2003.

11. You Y, Zhang J, Li Y, Li Y, Shi G, Ma L and Wei $\mathrm{H}$ CADM1/TSLC1 inhibits melanoma cell line A375 invasion through the suppression of matrix metalloproteinases. Mol Med Rep 10: 2621-2626, 2014.

12. He G, Lei W, Wang S, Xiao R, Guo K, Xia Y, Zhou X, Zhang K, Liu X and Wang Y: Overexpression of tumor suppressor TSLC1 by a survivin-regulated oncolytic adenovirus significantly inhibits hepatocellular carcinoma growth. J Cancer Res Clin Oncol 138: 657-670, 2012.
13. Yang G, He W, Cai M, Luo F, Kung H, Guan X, Zeng Y and Xie D: Loss/down-regulation of tumor suppressor in lung cancer 1 expression is associated with tumor progression and is a biomarker of poor prognosis in ovarian carcinoma. Int J Gynecol Cancer 21: 486-493, 2011.

14. Heller G, Geradts J, Ziegler B, Newsham I, Filipits M, Markis-Ritzinger EM, Kandioler D, Berger W, Stiglbauer W, Depisch D, et al: Downregulation of TSLC1 and DAL-1 expression occurs frequently in breast cancer. Breast Cancer Res Treat 103: 283-291, 2007.

15. Jansen M, Fukushima N, Rosty C, Walter K, Altink R, Heek TV, Hruban R, Offerhaus JG and Goggins M: Aberrant methylation of the $5^{\prime} \mathrm{CpG}$ island of TSLC1 is common in pancreatic ductal adenocarcinoma and is first manifest in high-grade PanlNs. Cancer Biol Ther 1: 293-296, 2002.

16. Takahashi Y, Iwai M, Kawai $T$, Arakawa A, Ito $T$, Sakurai-Yageta M, Ito A, Goto A, Saito M, Kasumi F and Murakami Y: Aberrant expression of tumor suppressors CADM1 and $4.1 \mathrm{~B}$ in invasive lesions of primary breast cancer. Breast Cancer 19: 242-252, 2012.

17. Lei W, Liu HB, Wang SB, Zhou XM, Zheng SD, Guo KN, Ma BY, Xia YL, Tan WS, Liu XY and Wang YG: Tumor suppressor in lung cancer-1 (TSLC1) mediated by dual-regulated oncolytic adenovirus exerts specific antitumor actions in a mouse model. Acta Pharmacol Sin 34: 531-540, 2013.

18. Lu B, Di W, Wang H, Ma H, Li J and Zhang Q: Tumor suppressor TSLC1 is implicated in cell proliferation, invasion and apoptosis in laryngeal squamous cell carcinoma by regulating Akt signaling pathway. Tumour Biol 33: 2007-2017, 2012.

19. Chen K, Wang G, Peng L, Liu S, Fu X, Zhou Y, Yu H, Li A, Li J, Zhang S, et al: CADM1/TSLC1 inactivation by promoter hypermethylation is a frequent event in colorectal carcinogenesis and correlates with late stages of the disease. Int J Cancer 128: 266-273, 2011

20. Fukuhara H, Kuramochi M, Fukami T, Kasahara K, Furuhata M, Nobukuni T, Maruyama T, Isogai K, Sekiya T, Shuin T, et al: Promoter methylation of TSLC1 and tumor suppression by its gene product in human prostate cancer. Jpn J Cancer Res 93: 605-609, 2002.

21. Ochiai H, Takenobu H, Nakagawa A, Yamaguchi Y, Kimura M, Ohira M, Okimoto Y, Fujimura Y, Koseki H, Kohno Y, et al: $\mathrm{Bmil}$ is a MYCN target gene that regulates tumorigenesis through repression of KIF1Bbeta and TSLC1 in neuroblastoma. Oncogene 29: 2681-2690, 2010.

22. Lung HL, Cheung AK, Xie D, Cheng Y, Kwong FM, Murakami Y, Guan XY, Sham JS, Chua D, Protopopov AI, et al: TSLC1 is a tumor suppressor gene associated with metastasis in nasopharyngeal carcinoma. Cancer Res 66: 9385-9392, 2006.

23. Hui AB, Lo KW, Kwong J, Lam EC, Chan SY, Chow LS, Chan AS, Teo PM and Huang DP: Epigenetic inactivation of TSLC1 gene in nasopharyngeal carcinoma. Mol Carcinog 38: 170-178, 2003.

24. Sacristan R, Gonzalez C, Fernández-Gómez JM, Fresno F, Escaf S and Sánchez-Carbayo M: Molecular classification of non-muscle-invasive bladder cancer (pTa low-grade, pT1 low-grade, and pT1 high-grade subgroups) using methylation of tumor-suppressor genes. J Mol Diagn 16: 564-572, 2014.

25. Agundez M, Grau L, Palou J, Algaba F, Villavicencio H and Sanchez-Carbayo M: Evaluation of the methylation status of tumour suppressor genes for predicting bacillus Calmette-Guérin response in patients with T1G3 high-risk bladder tumours. Eur Urol 60: 131-140, 2011.

26. Casadio V, Molinari C, Calistri D, Tebaldi M, Gunelli R, Serra L, Falcini F, Zingaretti C, Silvestrini R, Amadori D and Zoli W: DNA Methylation profiles as predictors of recurrence in non muscle invasive bladder cancer: An MS-MLPA approach. J Exp Clin Cancer Res 32: 94, 2013

27. Hellwinkel OJ, Kedia M, Isbarn H, Budäus L and Friedrich MG: Methylation of the TPEF- and PAX6-promoters is increased in early bladder cancer and in normal mucosa adjacent to pTa tumours. BJU Int 101: 753-757, 2008.

28. Eble JS, Epstein JI, Sesterhenn IA and Sauter G: Pathology and genetics of tumours of the urinary system and male genital organs. In: World Health Organization Classification of Tumours. Lyon: IARC Press; 2004.

29. Sobin LH, Gospodarowicz MK and Wittekind C: TNM classification of malignant tumours. 7th ed. Weinheim: Wiley; 2009.

30. Livak KJ and Schmittgen TD: Analysis of relative gene expression data using real-time quantitative PCR and the 2(-Delta Delta C(T)) method. Methods 25: 402-408, 2001. 
31. Mao X, Seidlitz E, Truant R, Hitt M and Ghosh HP: Re-expression of TSLC1 in a non-small-cell lung cancer cell line induces apoptosis and inhibits tumor growth. Oncogene 23: 5632-5642, 2004.

32. Qin L, Zhu W, Xu T, Hao Y, Zhang Z, Tian Y and Yang D: Effect of TSLC1 gene on proliferation, invasion and apoptosis of human hepatocellular carcinoma cell line HepG2. J Huazhong Univ Sci Technolog Med Sci 27: 535-537, 2007.

33. Cardif RD: Epithelial to mesenchymal transition tumors: Fallacious or snail's pace? Clin Cancer Res 11: 8534-8537, 2005.

34. Liang W, Hao Z, Han JL, Zhu DJ, Jin ZF and Xie WL: CAV-1 contributes to bladder cancer progression by inducing epithelial-to-mesenchymal transition. Urol Oncol 32: 855-863, 2014.

35. Wan F, Cheng C, Wang Z, Xiao X, Zeng H, Xing S, Chen X, Wang J, Li S, Zhang Y, et al: SATB1 overexpression regulates the development and progression in bladder cancer through EMT. PLoS One 10: e0117518, 2015.
36. Zhao J, Dong D, Sun L, Zhang G and Sun L: Prognostic significance of the epithelial-to-mesenchymal transition markers e-cadherin, vimentin and twist in bladder cancer. Int Braz J Urol 40: 179-189, 2014.

37. Geng J, Fan J, Ouyang Q, Zhang X, Zhang X, Yu J, Xu Z, Li Q, Yao X, Liu X and Zheng J: Loss of PPM1A expression enhances invasion and the epithelial-to-mesenchymal transition in bladder cancer by activating the TGF- $\beta /$ Smad signaling pathway. Oncotarget 5: 5700-5711, 2014.

38. Chhabra R: Cervical cancer stem cells: Opportunities and challenges. J Cancer Res Clin Oncol 141: 1889-1897, 2015.

(i) $($ This work is licensed under a Creative Commons Attribution-NonCommercial-NoDerivatives 4.0 International (CC BY-NC-ND 4.0) License. 